\title{
Characterization of piezoelectric polymer composites for MEMS devices
}

\author{
C V MADHUSUDHANA RAO and G PRASAD* \\ Department of Physics, Osmania University, Hyderabad 500 004, India
}

MS received 16 May 2009

\begin{abstract}
Composite piezoelectric ceramics are important materials for transducer applications in medical diagnostic devices and MEMS devices. In micrometer scale the material properties of piezopolymers or piezoceramics do not coincide with that of bulk materials. The present work is aimed at simulating the material properties of piezoceramics and piezo-polymer composite thin films in the micrometer scale and then to determine the piezo-composite material properties. Piezoceramics have very high electromechanical coupling coefficient $(k)$. But they have very high acoustic impedance and they are very brittle especially when thin films are fabricated. Piezopolymer like PVDF has low acoustic impedance and can be fabricated into thin films but it has very low $k$ value and high dielectric losses. The combination of piezoceramics and piezopolymers form the piezocomposites, which have suitable material properties for transducer applications. The composites can have different connectivities. For 2-2 composite, we can select two layers or a stack of PZT and PVDF layers. It is intended to determine the material properties both analytically and by simulation using computer simulation ANSYS software which implements finite element method (FEM). Although the simulation process presents approximate results, it can be verified from the large available experimental data from the literature with the simulated data.
\end{abstract}

Keywords. Piezocomposites; MEMS; finite element analysis.

\section{Introduction}

Ferroelectric ceramics and piezocomposites are widely used in many applications such as sensors, transducers and actuators used in MEMS devices. In such applications, bulk ferroelectric ceramic materials have certain shortcomings such as low fracture toughness, high inertia and a relatively large size. As a result the range of working frequency is limited due to brittleness, the dimensions are large in relation to the structure and adhesion of the piezoceramic to a structure is difficult due to different curvatures and surface roughness. For these reasons ferroelectric ceramics in thin films are employed in electromechanical devices such as MEMS as they have following advantages. Ferroelectric ceramic thin films have small inertia which allows for higher frequency applications, significantly higher dielectric strengths are obtained so that higher energy densities can be achieved along with relatively rapid polarization switching and require a relatively low driving voltage. The quality and reliability of MEMS device depends on the properties of ferroelectric thin films (Lian and Sottos 2000). They behave differently from bulk ferroelectric ceramics due to their planar geometry, high deposition temperature, high residual stress and different structure.

Although thin film PZT ceramic films can be employed in MEMS devices, their use is limited due to their brittleness and high acoustic impedance. For this reason piezoelectric polymers such as PVDF are considered as favourable

*Author for correspondence (gudurup@ osmania.ac.in) materials for micro-actuator applications due to their fast response, low operating voltage and greater efficiency of operation (Fu et al 2006). But the pieozpolymers have low electromechanical coupling coefficients and high dielectric losses.

For the above reasons, a piezocomposite which is a combination of piezoceramic (PZT) and piezopolymer (PVDF) is proposed for MEMS devices. These piezocomposites have suitable material properties such as moderate electromechanical coefficients, low acoustic impedance, low density, a wide range of dielectric constants, low dielectric loss and low mechanical loss. A variety of piezocomposite materials can be made by combining a piezoceramic with a passive polymer like epoxy or active polymer like PVDF. The pieozcomposites are classified according to their connectivity (such as $2-2,1-3,0-3$ etc). Connectivity is defined as the number of dimensions through which the material is continuous. It is conventional for the first digit to refer to the piezoelectrically active phase. Care should be taken while designing piezocomposite material because both the composition and physical dimensions affect the piezoelectric properties of composite.

The most commonly used method for designing composite transducer devices is the effective medium model proposed by Smith (1991). But the effective medium theory predicts higher resonance frequencies than the observed ones as reported elsewhere. For this reason FEM is adopted for both design and analysis of piezocomposite material properties which can be used in transducer design. Although the experimental results are much sought after, computer simulation 


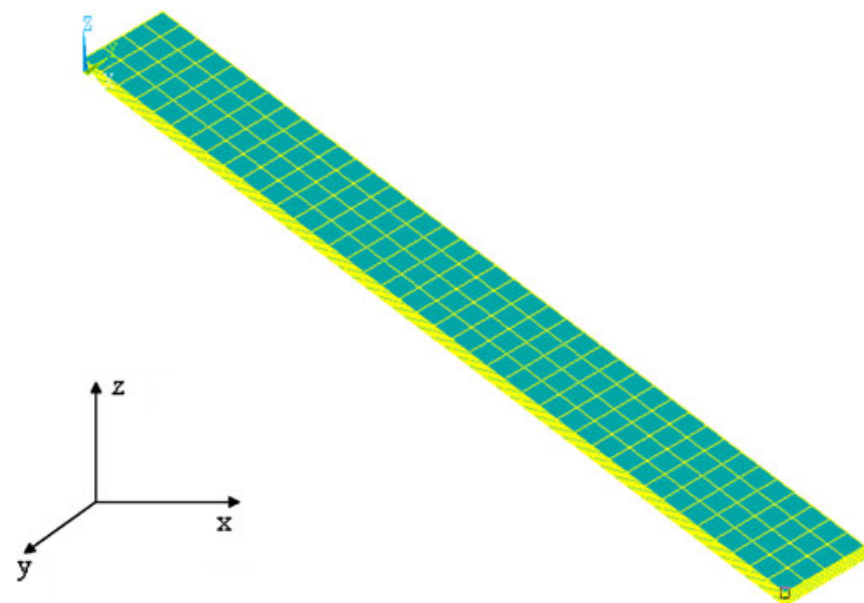

Figure 1. Cantilever beam with a selected node.

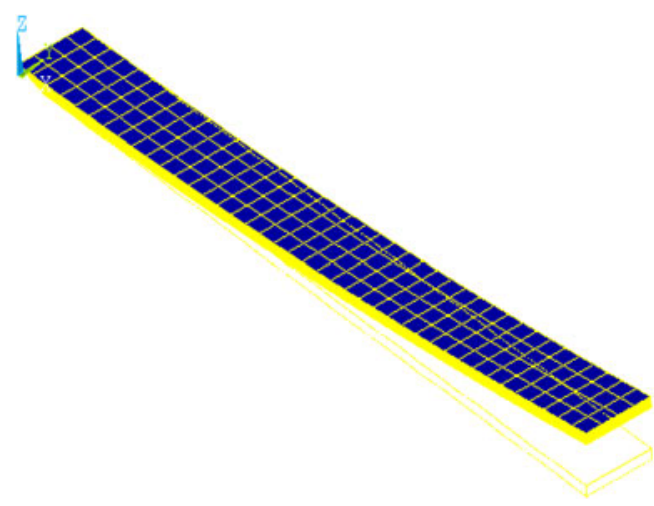

Figure 2. Vertical deflection of beam due to applied voltage.

analysis employing FEM is a good idea before the physical design of a complicated MEMS device.

\section{Finite element modeling}

At the first step, the sample piezoelectric material PZT-5A is taken in the form of a thin film of length, $5 \mathrm{~mm}$, width, $1 \mathrm{~mm}$ and thickness, 1 micrometer on nickel substrate of thickness, $5 \mu \mathrm{m}$, with same length and width as the piezoelectric film, in accordance with IEEE standards (ANSI/IEEE 1987). The length, width and thickness of the beam are taken along $X$, $Y$ and $Z$-axes, respectively as shown in figure 1 . The 8 -node
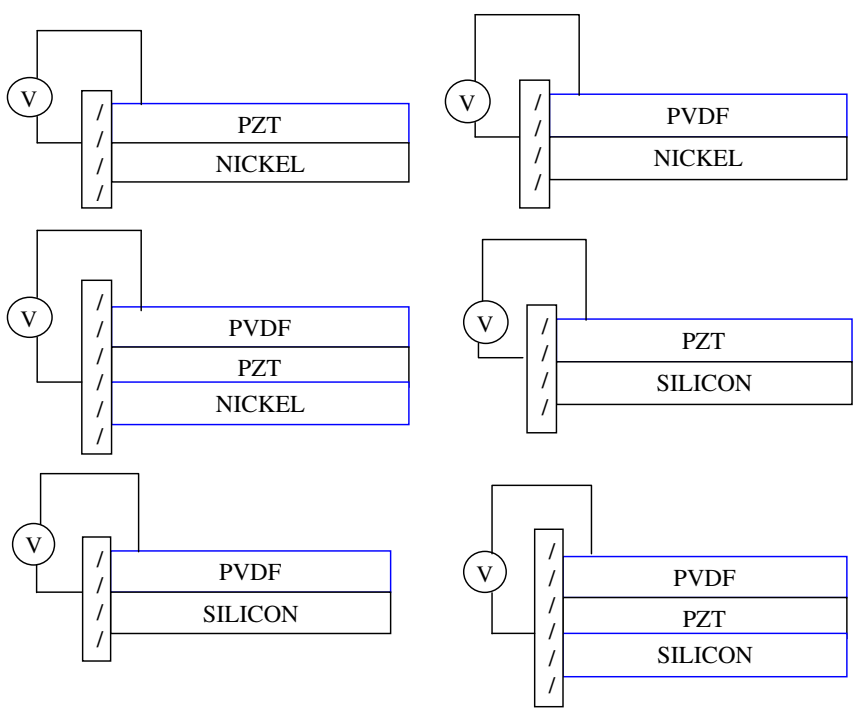

Figure 3. PZT and PVDF films on nickel substrate and silicon substrate.

brick element for piezoelectric solid is defined by coupled field piezoelectric element SOLID5 and SOLID45 element is defined for structural solid nickel substrate. The entire volume is divided into finite number of elements by generating the mesh. In this process nodes are formed (figure 2).

The nodal degrees of freedom (DOF) are UX ( $x$ displacement), UY ( $y$ displacement), UZ ( $z$ displacement), and VOLT (electrical potential). Constraints are applied on the displacements at one end to fix the beam and it is free to vibrate at the other end so that it can be treated as a cantilever beam. Loads in the form of voltages are applied at the nodes. Material properties are defined for each material which is tabulated in table 1. It should be noted that the thin film PZT piezoelectric coefficient is different from its bulk value (Lian and Sottos 2000). The polarization axis is chosen along $Z$-axis. The nodes on the top and the bottom faces of the beam are coupled and the bottom face is grounded. The top face is given a voltage. The resulting displacements along $Z$-axis are taken. This type of solid modeling takes much processing time to obtain the results. Therefore, this is simplified by replacing solid modeling with plane modeling by taking PLANE223 which is a 2-dimensional 8-node coupled field solid element. For the substrate PLANE42 which is a 2-dimensional 4-node structural solid element is taken. The model beams with nickel and silicon substrates are shown in figure 3 .

Table 1. Material properties.

\begin{tabular}{lccccccc}
\hline $\begin{array}{l}\text { Material/ } \\
\text { Property }\end{array}$ & $Y(\mathrm{GPa})$ & $G(\mathrm{GPa})$ & $v$ & $\rho\left(\mathrm{kg} / \mathrm{m}^{3}\right)$ & $d_{31}(\mathrm{pC} / \mathrm{N})$ & $d_{33}(\mathrm{pC} / \mathrm{N})$ & $K=\varepsilon_{33} / \varepsilon_{0}$ \\
\hline PZT $(1 \mu \mathrm{m})$ & 69 & $23 \cdot 26$ & $0 \cdot 3$ & 7800 & -33.9 & 25 & 540 \\
PZT $(2 \mu \mathrm{m})$ & 69 & $23 \cdot 26$ & $0 \cdot 3$ & 7800 & $-33 \cdot 9$ & 46 & 735 \\
PVDF & $2 \cdot 8$ & $0 \cdot 8$ & 0.29 & 1800 & 25 & -34 & 12 \\
Ni & 150 & 56.4 & 0.33 & 8900 & - & - & - \\
$\mathrm{Si}$ & 120 & $42 \cdot 25$ & 0.42 & 2329 & - & - & - \\
\hline
\end{tabular}



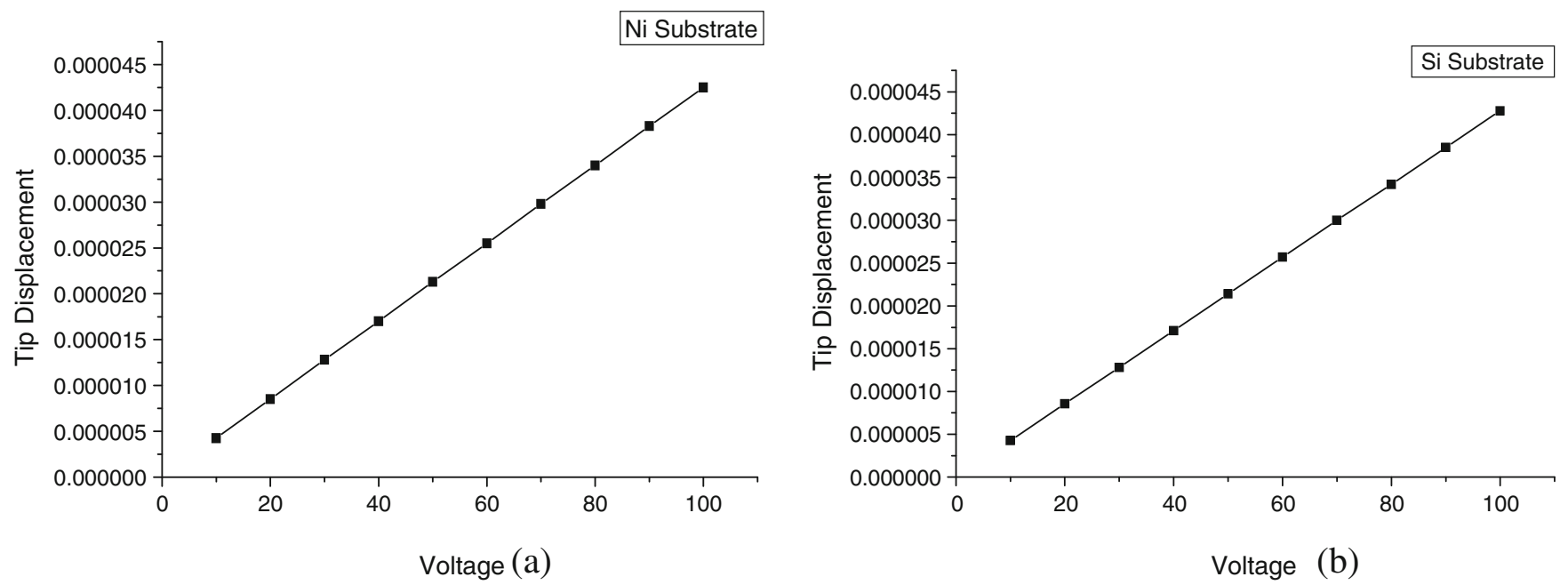

Figure 4. Tip displacement of composite with (a) nickel substrate and (b) silicon substrate.

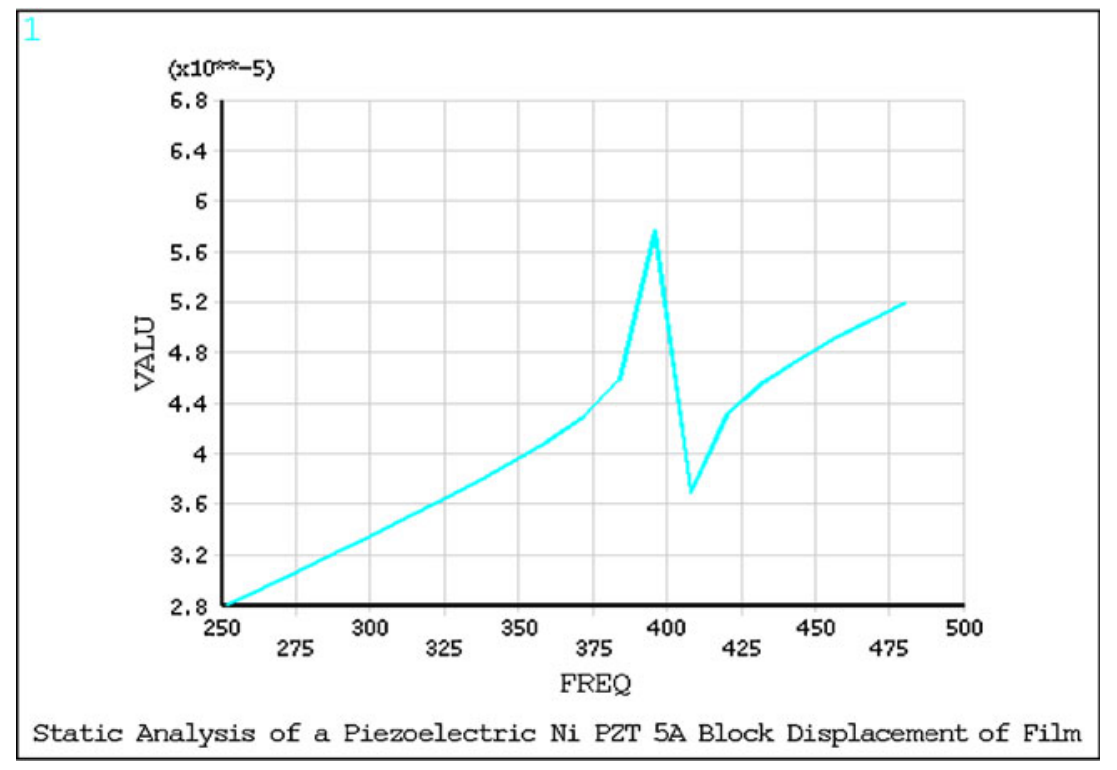

Figure 5. Admittance vs frequency for Ni-PZT $(1 \mu \mathrm{m})-\mathrm{PVDF}$ composite beam.

\section{Finite element analysis}

The piezoelectric ceramic, PZT, on nickel substrate is fabricated in the form of composite cantilever beam and it is subjected to static, modal and harmonic analysis by ANSYS software. The static analysis is done by applying a steady voltage of $100 \mathrm{~V}$ between the top and bottom faces with the electrodes on the top and bottom surfaces of the sample. The tip deflection of the beam along $Z$-axis $(\delta)$ is shown in figure 2. It can be calculated from the following (1) as given in (Devoe and Pisano 1997)

$$
\delta=\frac{3 t_{\mathrm{e}}\left(t_{\mathrm{e}}+t_{\mathrm{p}}\right) E_{\mathrm{e}} E_{\mathrm{p}} L^{2} d_{31} V}{E_{\mathrm{e}}^{2} t_{\mathrm{e}}^{4}+E_{\mathrm{e}} E_{\mathrm{p}}\left(4 t_{\mathrm{e}}^{3} t_{\mathrm{p}}+6 t_{\mathrm{e}}^{2} t_{\mathrm{p}}^{2}+4 t_{\mathrm{e}} t_{\mathrm{p}}^{3}\right)+E_{\mathrm{p}}^{2} t_{\mathrm{p}}^{4}},
$$

where $t_{\mathrm{e}}$ and $t_{\mathrm{p}}$ are thickness of substrate and piezoelectric material, respectively. $E_{\mathrm{e}}$ and $E_{\mathrm{p}}$ are Young's modulus of substrate and piezoelectric material, respectively. $L$ is the length of the beam, $d_{31}$ the transverse piezoelectric coefficient and $V$ the applied voltage. For multilayer cantilever beam, the equation for tip deflection is given by (2) as given in (Wang and Cross 1999)

$$
\delta=\frac{-3}{E_{\text {eq }} h_{\text {eq }}^{2}} \frac{E_{\mathrm{f}}\left(E_{\mathrm{s}} h_{\mathrm{s}}+E_{\mathrm{e}} h_{\mathrm{e}}\right)}{E_{\mathrm{s}} h_{\mathrm{s}}+E_{\mathrm{f}} h_{\mathrm{f}}+E_{\mathrm{e}} h_{\mathrm{e}}} d_{31} L^{2} V,
$$

where metal substrate (s), PZT film (f) and electrodes (e) have thickness $h_{\mathrm{s}}, h_{\mathrm{f}}$ and $h_{\mathrm{e}}$, respectively. $L$ represents the cantilever's length. The equivalent thickness,

$$
h_{\mathrm{eq}}=h_{\mathrm{s}}+h_{\mathrm{f}}+h_{\mathrm{e}} \text {, }
$$

while $E_{\mathrm{eq}}$ represents the equivalent elastic modulus that can be written as

$$
E_{\mathrm{eq}}=\frac{K_{1}+K_{2}+K_{3}+K_{4}+K_{5}}{\left(E_{\mathrm{f}} h_{\mathrm{f}}+E_{\mathrm{s}} h_{\mathrm{s}}+E_{\mathrm{e}} h_{\mathrm{e}}\right) h_{\mathrm{eq}}^{3}}
$$




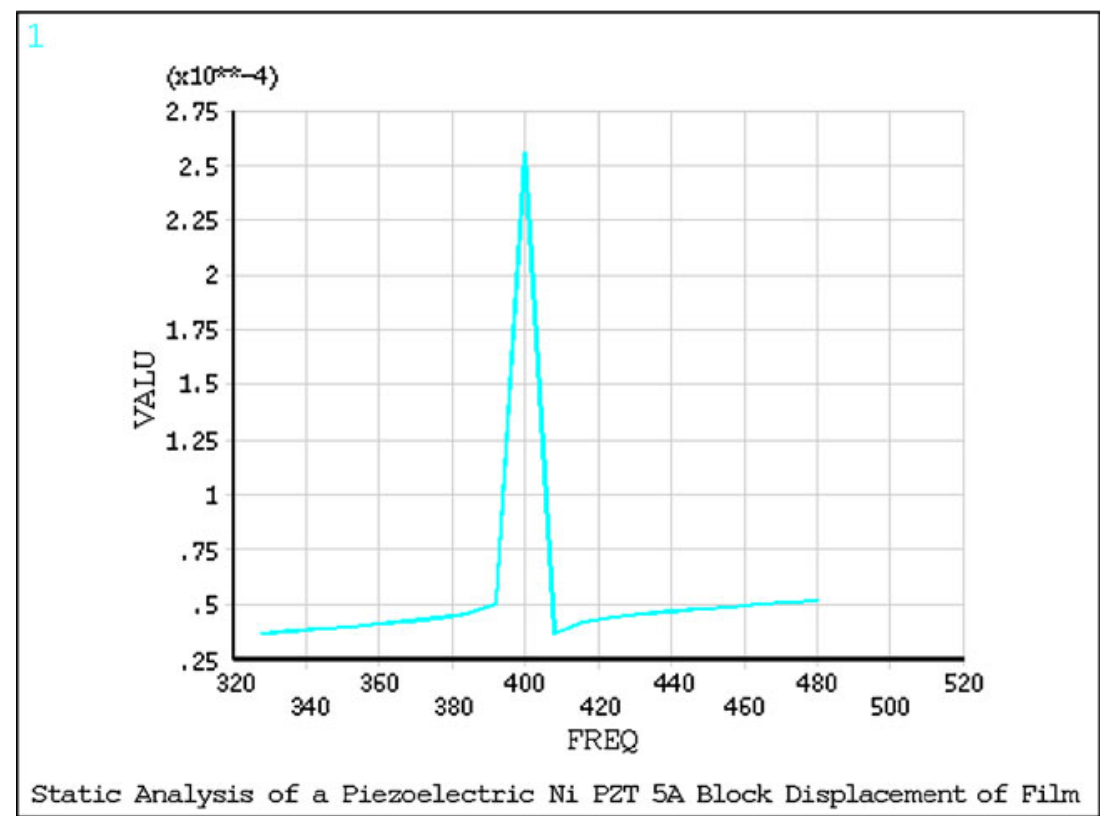

Figure 6. Admittance vs frequency for Ni-PZT $(2 \mu \mathrm{m})-\mathrm{PVDF}$ composite beam.

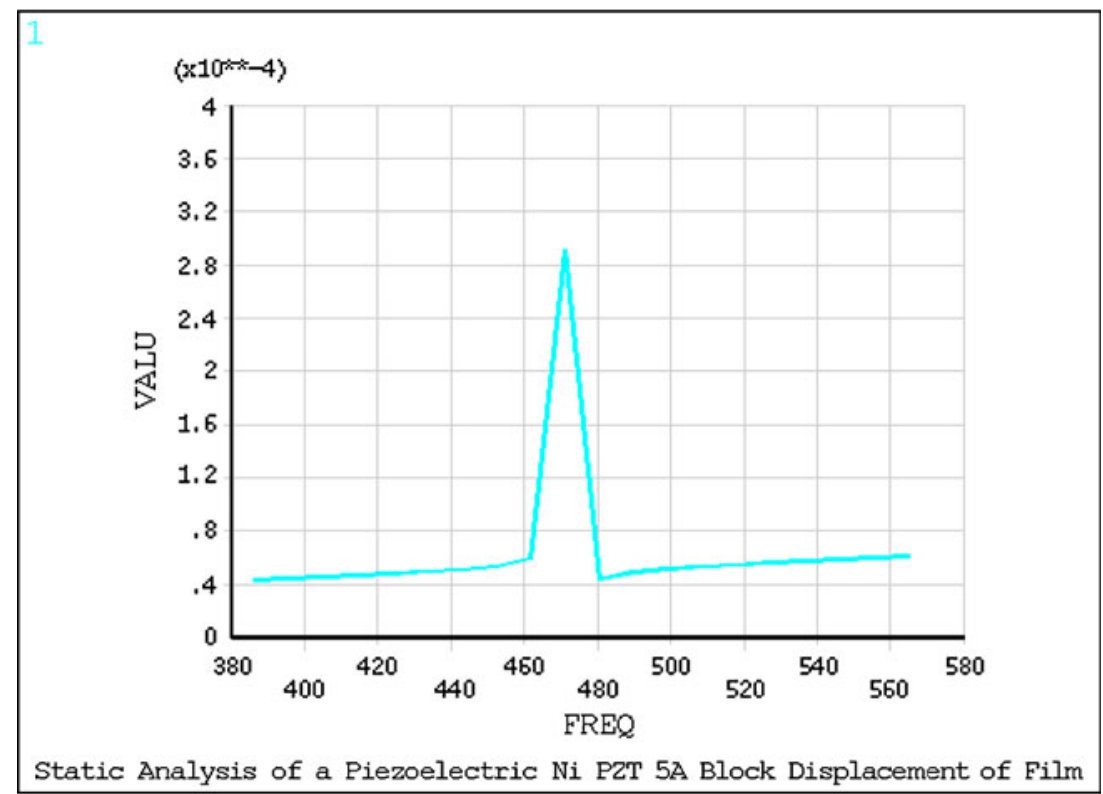

Figure 7. Admittance vs frequency for Si-PZT(1 $\mu \mathrm{m})-\mathrm{PVDF}$ composite beam.

with

$$
\begin{aligned}
& K_{1}=E_{\mathrm{f}}^{2} h_{\mathrm{f}}^{4}+E_{\mathrm{s}}^{2} h_{\mathrm{s}}^{4}+E_{\mathrm{e}}^{2} h_{\mathrm{e}}^{4} \\
& K_{2}=2 E_{\mathrm{s}} E_{\mathrm{e}} h_{\mathrm{s}} h_{\mathrm{e}}\left(2 h_{\mathrm{s}}^{2}+2 h_{\mathrm{e}}^{2}+3 h_{\mathrm{s}} h_{\mathrm{e}}\right), \\
& K_{3}=2 E_{\mathrm{f}} E_{\mathrm{e}} h_{\mathrm{f}} h_{\mathrm{e}}\left(2 h_{\mathrm{f}}^{2}+2 h_{\mathrm{e}}^{2}+3 h_{\mathrm{f}} h_{\mathrm{e}}\right), \\
& K_{4}=2 E_{\mathrm{f}} E_{\mathrm{e}} h_{\mathrm{f}} h_{\mathrm{e}}\left(2 h_{\mathrm{f}}^{2}+2 h_{\mathrm{e}}^{2}+3 h_{\mathrm{f}} h_{\mathrm{e}}\right), \\
& K_{5}=12 E_{\mathrm{s}} E_{\mathrm{f}} h_{\mathrm{s}} h_{\mathrm{f}} h_{\mathrm{e}} h_{\mathrm{eq}} .
\end{aligned}
$$

As there exists a linear relation between $\delta$ and $V$, tip deflection for various values of applied voltage is plotted and $d_{31}$ is determined from the slope of the graph (figure 4).

The vertical displacement of the beam along $Z$-axis at different segments of the beam is noted from figure 2. It can be observed that the vertical displacement increases from the fixed end to the free end of the beam. The beam is then subjected to modal analysis. Ten modes are extracted for each sample. The results of modal analysis are used in harmonic analysis to analyse the harmonic response of the sample on applying a voltage of $100 \mathrm{~V}$. 


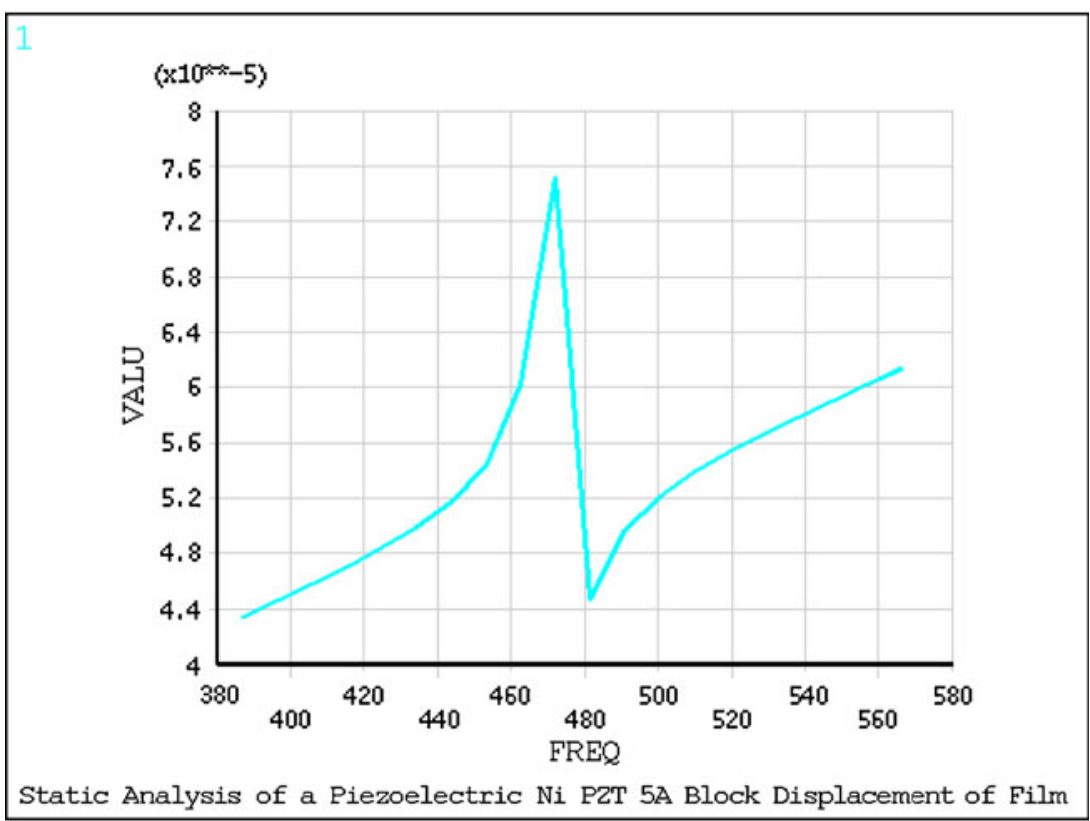

Figure 8. Admittance vs frequency for Si-PZT $(2 \mu \mathrm{m})-\mathrm{PVDF}$ composite beam.

Table 2. Pertinent data.

\begin{tabular}{|c|c|c|c|c|c|c|c|c|}
\hline \multirow[b]{2}{*}{ Substrate } & \multirow[b]{2}{*}{ Piezo material } & \multicolumn{2}{|c|}{ Tip Disp $(\mu \mathrm{m})$} & \multirow[b]{2}{*}{$d_{31}(\mathrm{pC} / \mathrm{N})$} & \multicolumn{3}{|c|}{ Frequency $(\mathrm{Hz})$} & \multirow[b]{2}{*}{$k_{31}$} \\
\hline & & Theory & FEM & & First mode & $f_{\mathrm{r}}$ & $f_{\mathrm{a}}$ & \\
\hline Nickel & PVDF & 44.42 & 44.59 & -25 & 592 & 591 & 615 & $0 \cdot 31$ \\
\hline Nickel & PZT $(1 \mu \mathrm{m})$ & 3800 & 3750 & $-33 \cdot 9$ & 143 & 142 & 149 & 0.34 \\
\hline Nickel & PZT $(2 \mu \mathrm{m})$ & 2800 & 2840 & $-33 \cdot 9$ & 159 & 158 & 165 & 0.32 \\
\hline Nickel & Composite (3\%) & 42.74 & $42 \cdot 53$ & $-25 \cdot 15$ & 400 & 395 & 410 & $0 \cdot 30$ \\
\hline Nickel & Composite (6\%) & $40 \cdot 98$ & $40 \cdot 65$ & $-33 \cdot 9$ & 404 & 403 & 413 & $0 \cdot 24$ \\
\hline Silicon & PVDF & $44 \cdot 6$ & 44.41 & -25 & 1142 & 1136 & 1168 & $0 \cdot 26$ \\
\hline Silicon & $\mathrm{PZT}(1 \mu \mathrm{m})$ & 4300 & 4330 & -33.9 & 216 & 215 & 220 & $0 \cdot 24$ \\
\hline Silicon & $\mathrm{PZT}(2 \mu \mathrm{m})$ & 3100 & 3110 & -33.9 & 222 & 218 & 223 & 0.23 \\
\hline Silicon & Composite (3\%) & $43 \cdot 14$ & 42.79 & $-25 \cdot 09$ & 474 & 472 & 484 & $0 \cdot 25$ \\
\hline Silicon & Composite (6\%) & $41 \cdot 58$ & $41 \cdot 01$ & -33.9 & 471 & 470 & 480 & $0 \cdot 23$ \\
\hline
\end{tabular}

The electrical and mechanical responses of the material near the resonance and anti-resonance frequencies is studied. Admittance vs frequency graph is shown in figures 5-8 from which resonance and anti-resonance frequencies are obtained. Resonance frequency corresponds to maximum admittance value and anti-resonance corresponds to minimum admittance value.

The variation of electrical impedance with frequency is studied. The electromechanical coupling coefficient $\left(k_{31}\right)$ is calculated from the following equation

$$
k_{31}^{2}=\frac{\left.(\pi / 2)\left(f_{\mathrm{a}} / f_{\mathrm{r}}\right) \tan \left[(\pi / 2)\left[\left(f_{\mathrm{a}}-f_{\mathrm{r}}\right) / f_{\mathrm{r}}\right)\right]\right]}{\left.1+(\pi / 2)\left(f_{\mathrm{a}} / f_{\mathrm{r}}\right) \tan \left[(\pi / 2)\left[\left(f_{\mathrm{a}}-f_{\mathrm{r}}\right) / f_{\mathrm{r}}\right)\right]\right]},
$$

where $f_{\mathrm{r}}$ and $f_{\mathrm{a}}$ are the resonance and anti-resonance frequencies, respectively around the first natural mode frequency.
The same procedure is followed to analyse the individual composite beams of PZT of $2 \mu \mathrm{m}$ thick and PVDF of $30 \mu \mathrm{m}$ thick on nickel substrate and the results are tabulated. Finally, a piezocomposite beam with $2-2$ connectivity is fabricated on nickel substrate with PZT film of thickness, $1 \mu \mathrm{m}$ and PVDF of $30 \mu \mathrm{m}$ thick which results in a PZT volume fraction of $3 \%$. Similarly a PZT volume fraction of $6 \%$ is achieved by increasing PZT thickness to $2 \mu \mathrm{m}$. The results are tabulated in table 2.

Both the piezocomposite beams are studied to analyse their properties. It is found that the deflection is found to decrease due to the piezocomposite film as compared with that of pure PZT film. But due to the presence of polymer it has better mechanical flexibility and better impedance matching along with suitable coupling coefficients. The first natural frequency of the piezocomposite beam is increased due to the presence of polymer. But the resonance and 
anti-resonance frequencies are stretched over longer frequency region as compared to that of pure PZT film where the resonance and anti-resonance frequencies are sharply defined. This results in a broader bandwidth. Similar analysis is done by replacing the nickel substrate with the silicon substrate of same thickness for comparative study.

\section{Conclusions}

Thin piezoelectric materials in the form of cantilever beam are modeled in finite element form and they are subjected to static, modal and harmonic analysis by ANSYS software. Various resonance and anti-resonance frequencies are obtained for each material. The electromechanical coefficients are calculated for each set of resonance and anti- resonance frequencies. Comparative study is done for materials properties of thin piezocomposite of various compositions which show better electromechanical properties than a pure piezoceramic or a pure piezopolymer.

\section{References}

ANSI/IEEE Std 176-1987 IEEE standard on piezoelectricity (New York: The Institute of Electrical and Electronics Engineers)

Devoe D L and Pisano A 1997 J. Microelectromech. S. 6266

Fu Yao, Harvey Erol C, Ghantasala M K and Spinks Geoff 2006 Smart Mater. Struct. 15 S141-S146

Lian L and Sottos N R 2000 J. Appl. Phys. 873941

Smith W A 1991 IEEE Trans. Ultrason. Ferr. 3840

Wang Q M and Cross L E 1999 IEEE Trans. Ultrason. Ferr. 46 1343 